\title{
New Onset Obsessive Compulsive Disorder Following High Frequency Repetitive Transcranial Magnetic Stimulation over Left Dorsolateral Prefrontal Cortex for Treatment of Negative Symptoms in a Patient with Schizophrenia
}

\author{
Harshit Garg, Saurabh Kumar, Swarndeep Singh, Nand Kumar, Rohit Verma \\ Department of Psychiatry, All India Institute of Medical Sciences, New Delhi, India
}

\begin{abstract}
Obsessive-compulsive symptoms and/or obsessive-compulsive disorders (OCD) are frequently comorbid with schizophrenia, though the exact clinical and etiological relationship between them is poorly understood. Here we describe a case that, to the best of our knowledge, is the first report of new-onset OCD in a patient who was receiving high-frequency repetitive transcranial magnetic stimulation over left dorsolateral pre-frontal cortex as an adjuvant therapy for negative symptoms of schizophrenia. Thisreport supports our understanding of OCD as a brain disorder involving hyperactivity of pre-frontal cortex and cortico-striatal-thalamo-cortical circuit dysfunction.
\end{abstract}

KEY WORDS: Obsessive-compulsive disorder; Negative symptoms; Schizophrenia; Transcranial magnetic stimulation.

\section{INTRODUCTION}

Obsessive-compulsive symptoms (OCS) and/or obsessive-compulsive disorders (OCD) are frequently found in patients with schizophrenia with rates as high as $30 \% .^{1}$ Neuroimaging studies have shown that hypofrontality and lack of dopamine in prefrontal cortex may be related with negative symptoms of schizophrenia. ${ }^{2)}$ Based on this hypothesis high-frequency repetitive transcranial magnetic stimulation (rTMS) shown to increase cortical excitability and modulate dopamine release, has been used as a possible adjuvant therapy for negative symptoms of schizophrenia. ${ }^{3)}$ Whereas, dysfunctional cortico-striatothalamo-cortical (CSTC) loops have been implicated as one of the possible mechanism for causing symptoms of OCD. ${ }^{4)}$ The hyperactivity in orbitofrontal cortex, anterior cingulate cortex and caudate nucleus have been linked to

Received: February 1, 2018 / Revised: February 23, 2018 Accepted: March 4, 2018

Address for correspondence: Rohit Verma

Department of Psychiatry, All India Institute of Medical Sciences, Fourth Floor, Teaching Block, Ansari Nagar, New Delhi, NCT-Delhi 110029, India

E-mail: drrv321@gmail.com

ORCID: https://orcid.org/0000-0001-7418-2511
OCD, and the surgical resection of areas involving this circuit relieved OCD, i.e., overdriven CSTC circuit. Although several hypotheses have been postulated but the exact clinical and etiological relationship between schizophrenia and OCD is poorly understood. Here we describe a case that, to the best of our knowledge, is the first report of new-onset OCD in a patient who was receiving high-frequency rTMS over left dorsolateral prefrontal cortex (Lt-DLPFC) as an adjuvant therapy for negative symptoms of schizophrenia.

\section{CASE}

We report a case of an 18-year-old female, with no past or family history of psychiatric or neurological disorder, suffering from schizophrenia (according to ICD-10 [10th revision of the International Statistical Classification of Diseases and Related Health Problems] criteria) since the age of around 10 years. Her illness started with symptoms of delusion of persecution and third-person auditory hallucinations, which improved after starting treatment with risperidone up to $8 \mathrm{mg} /$ day and trihexyphenidyl (4 $\mathrm{mg} /$ day) at age of around 15 years. Her positive symptoms were adequately controlled, and negative symptoms in

(ㄷ) This is an Open-Access article distributed under the terms of the Creative Commons Attribution Non-Commercial License (http://creativecommons.org/licenses/by-nc/4.0) which permits unrestricted non-commercial use, distribution, and reproduction in any medium, provided the original work is properly cited. 
the form of avolition, apathy, anhedonia, impaired attention-concentration and impaired self-care emerged despite of compliance to above treatment regimen for about past 2 years. Subsequently, risperidone and trihexyphenidyl were cross tapered with olanzapine (up to $20 \mathrm{mg} /$ day) from about past one year. Though, her positive symptoms ameliorated, above described residual negative symptoms persisted resulting in significant socio-occupational dysfunction. There was no history of significant head injury, loss of consciousness, seizures, and no family history of psychiatric or neurological disorder among first-degree relatives. Subsequently, she was referred to rTMS clinic, department of psychiatry for detailed assessment and management of negative symptoms with adjuvant rTMS. Detailed interview and clinical examination by a psychiatrist, and routine investigations along with thyroid function test revealed no psychiatric or medical comorbidity. She did not have any significant extrapyramidal symptoms. She was offered adjuvant rTMS as a potential treatment strategy for relief of negative symptoms. Her scores of Positive and Negative Syndrome Scale (PANSS) and Scale for the Assessment of Negative Symptoms (SANS) were 53 and 58, respectively at baseline assessment (prior to starting rTMS).

After obtaining informed written consent from patient, high-frequency rTMS in accordance with the updated safety guidelines, ${ }^{5)}$ was administered using Magstim Rapid device (Magstim, Whitland, UK) with a 70-mm figure-ofeight air-film coil. Stimulation parameters of $20-\mathrm{Hz}$ frequency at $100 \%$ of resting motor threshold intensity, with 10 seconds train duration, inter-train interval of $90 \mathrm{sec}-$ onds, and a total 2,000 pulses/session were used. A total of 20 sessions of rTMS, five days per week (from Monday to Friday) over a period of four weeks were given. The coil was placed over the Lt DLPFC, as per the standard procedure, determined by moving the TMS coil $5.0 \mathrm{~cm}$ rostrally from the right thumb area. ${ }^{6)}$ There was significant improvement in her negative symptoms, with PANSS and SANS scores decreased to 44 and 41 respectively.

She started remaining overtly concerned about her hygiene after receiving about 18 rTMS sessions. She would wash her hands/feet repeatedly and change clothes after returning from outside unlike previously, stating that they had become dirty. These symptoms were first reported by caregiver, five days after the completion of 20th rTMS session. A detailed psychiatric interview revealed new- onset obsessive doubts of contamination with compulsive washing and avoidance of contact with unknown people in the patient. These obsessive-compulsive symptoms were an additionally significant morbidity to her ongoing negative symptoms which further worsened her dysfunction. Hence, an ICD-10 diagnosis of OCD was made and Yale-Brown Obsessive-Compulsive Scale (YBOCS) was applied (score of 25). She was started on fluoxetine (up to $40 \mathrm{mg} /$ day) after two weeks (to allow for spontaneous resolution of symptoms) due to persistence of these symptoms, with which there was significant improvement in her OCD symptoms over a period of 2 months. She continues to maintain well six-month post-rTMS treatment and is in active follow-up. The improvement in both negative and OCD symptoms is maintained with her SANS and YBOCS scores of 40 and 7, respectively, at last assessment conducted six-months after last rTMS session. She has started participating in some household chores and her attention and concentration has also improved.

\section{DISCUSSION}

The patient presented above had no history of pre-existing OCS/OCD and did not report any OCS/OCD while she was maintaining on same dose of Olanzapine for about a year prior to starting of rTMS treatment. Her OCS started towards the end of rTMS treatment course, with no medication changes made before and during rTMS; making medication-related OCD unlikely. Magnetic resonance imaging of brain and electroencephalography revealed no abnormality. Thus, a clinical diagnosis of TMSrelated OCD was made for this event. However, there is a minor possibility of patient developing OCD symptoms independent of the rTMS treatment received.

Neurobiological studies support the proposition that abnormal activation patterns of the frontal-striato-thalamo-cortical circuit play a major role in generation of OCD symptoms, specifically the hyperactivity of pre-frontal cortex. ${ }^{7)}$ Studies have reported, positive correlation between washing and cleaning OCD symptoms and prefrontal cortex hyperactivity. ${ }^{8)}$ Further, low-frequency rTMS has been shown to reduce the fronto-striatal hyper-connectivity, which correlated with the degree of symptomatic improvement in patients with OCD. ${ }^{5)}$ We hypothesize that high-frequency rTMS given Lt-DLPFC can result in increased fronto-striatal connectivity precipitating 
OCD in vulnerable individuals.

However, the neurobiology of both OCD and schizophrenia involve complex interface between dopamine and serotonin neurotransmitter systems, in addition to above described neuromodulatory systems. ${ }^{9)}$ There is a lack of consensus about the neurobiology and management of OCS/OCD in schizophrenia and our case report aims to guide researchers and clinicians in planning future controlled studies assessing the pathogenesis and treatment of OCS/OCD in schizophrenia.

This case is important because it is the first report of OCD occurring after rTMS treatment. The presentation is consistent with our understanding of OCD as a brain disorder involving hyperactivity of pre-frontal cortex and cortico-striatal-thalamo-cortical circuit dysfunction.

\section{Conflicts of Interest}

No potential conflict of interest relevant to this article was reported.

\section{REFERENCES}

1. Byerly M, Goodman W, Acholonu W, Bugno R, Rush AJ. Obsessive compulsive symptoms in schizophrenia: frequency and clinical features. Schizophr Res 2005;76:309-316.

2. Hill K, Mann L, Laws KR, Stephenson CM, Nimmo-Smith I, McKenna PJ. Hypofrontality in schizophrenia: a meta-analy- sis of functional imaging studies. Acta Psychiatr Scand 2004; 110:243-256.

3. Shi C, Yu X, Cheung EF, Shum DH, Chan RC. Revisiting the therapeutic effect of rTMS on negative symptoms in schizophrenia: a meta-analysis. Psychiatry Res 2014,215:505-513.

4. Velikova S, Locatelli M, Insacco C, Smeraldi E, Comi G, Leocani L. Dysfunctional brain circuitry in obsessive-compulsive disorder: source and coherence analysis of EEG rhythms. Neuroimage 2010;49:977-983.

5. Rossi S, Hallett M, Rossini PM, Pascual-Leone A; Safety of TMS Consensus Group. Safety, ethical considerations, and application guidelines for the use of transcranial magnetic stimulation in clinical practice and research. Clin Neurophysiol 2009;120:2008-2039.

6. Pascual-Leone A, Rubio B, Pallardó F, Catalá MD. Rapid-rate transcranial magnetic stimulation of left dorsolateral prefrontal cortex in drug-resistant depression. Lancet 1996;348: 233-237.

7. Rosenberg DR, Keshavan MS. A.E. Bennett Research Award. Toward a neurodevelopmental model of of obsessive--compulsive disorder. Biol Psychiatry 1998;43:623-640.

8. Dunlop K, Woodside B, Olmsted M, Colton P, Giacobbe P, Downar J. Reductions in cortico-striatal hyperconnectivity accompany successful treatment of obsessive-compulsive disorder with dorsomedial prefrontal rTMS. Neuropsychopharmacology 2016;41:1395-1403.

9. Hwang MY, Yum SY, Kwon JS. Schizophrenia with obsessive-compulsive features: review of clinical and conceptual issues. Clin Psychopharmacol Neurosci 2009;7:9-14. 